\title{
Karunungang-bayang Irosanon: Isang pagtalunton
}

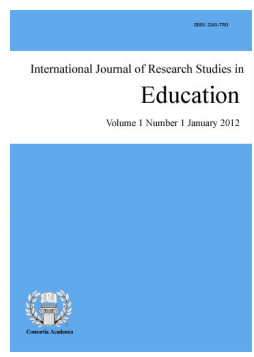

Llaneta, Rolly N. $\triangle$

Gallanosa National High School

Sorsogon State College, Philippines (Rolandoneo547@gmail.com)

Received: 1 September 2020

\section{Abstract}

This study aims to know and to measure the different folklore as part of culture and literature of Irosanons. The objectives of the study are the following: First to know the different folklore in the town of Irosin .Second to make sure if still there are preserved and enriched folklores by Irosanons. Third is to determine the use and advantage of folklores in the lives of citizens. It is also expected in this study to suggest essential activity based from the result of the study. The descriptive analysis method was used by the researcher; the data gathered were analyzed, checked and given statistical interpretation such as ranking and frequency count. The respondents were citizens of Irosin with knowledge about folklores. This study discovered that there were still different preserved and enriched folklores in the town of Irosin until this present time. These folklores have different uses and advantages. The intervention or activity made can be suggested in order to give importance to the culture and literature. A book was created by the researcher about the folklores of Irosin. It was recommended in the study to encourage students and the community to read books in order to understand more about the remaining folklore of the town and to integrate this literature that includes cultural theme in teaching. The local government should enact contests which enrich more the Filipino culture.

Keywords: folklore; Irosanon literature; tracing 


\section{Karunungang-bayang Irosanon: Isang pagtalunton}

\section{Introduksyon}

Sa panahong hindi pa nasasakop ng mga dayuhan ang Pilipinas nagtatag na ito ng sariling pamahalaan na tinatawag na balangay o barangay. Sa kadahilanang nakatayo na ang mga sinaunang tao o ninuno ng kanilang sariling paa sa pamamahala, mayroon na silang sariling batas. Bukod pa doon mayroon na rin silang pananampalataya, sining sa anyo ng panitikan at wika na pinatunayan ni Padre Pedro Chirino, isang Kastilang mananalaysay sa kanyang Relacion de las Islas Filipinas (1604). Mayaman sa kuwentong-bayan ang Pilipinas kaya sa panahon ng kuwentong-bayan napabilang din ang iba pang uri ng panitikan tulad ng kantahing-bayan, karunungang bayan at bulong ayon kay Rubin et al. (2006).

Noon, pa man bago dumating ang mga mananakop sa taong 1300 AD ang mga Aeta ay may sarili ng karunungang-bayan (Longga, 2010). Ang karunungang-bayan ay bahagi na ng kulturang Pilipino kaalinsabay ng pag-usbong ng sibilisasyon. Bahagi ito ng kasaysayan na may layuning makapagbigay ng karunungan sa mga mamamayan, at kasangkapaan rin ito sa pagpasalin-salin ng tradisyon at kultura ng bansa.

Ang kultura ay gintong pamana sa mga Pilipino ng mga ninuno nito na kailangang panatilihin at pagyamanin. Ito rin ang nagdudugtong ng pamumuhay ng mga tao mula sa nakaraan tungo sa kasalukuyan at nagsisilbing pagkakakilanlan ng isang lahi. Ang panitikan at kultura ay may kaugnayan sa isa't isa sapagkat lilipas man ang panahon, hindi maitatatwa ng isang lahi na naging kasangkapan ang mga ito upang mapaunlad ang kalinangan at repleksyon ang pantikan sa kultura na kapwa magagamit ng mga susunod na salinlahi. Ang kultura ay ang mga pananampalataya, mga tradisyon at kahalagahan (values) at iba pang gawain ng partikular na mga grupo ng tao sa isang partikular na panahon. Ang mga gawa ng panitikan ay mga tiyak na mabuting indikasyon ng mga kahalagahan (values) ng mga tao. Tiningnan ng panitikan ang kultura nang malaliman.

Ayon kay Uriarte (2019) ang karunungang bayan ay isang sangay ng panitikan na nagiging daan upang maipahayag ang mga kaisipan na napapabilang sa kultura ng bawat tribo. Ito ay may kahalagahan sa katutubong tradisyon na magiging gabay sa pagbasa at pagpapanitik sa anumang wika na naisulat ito, sa anumang panahon sa pananaw ng isang Pilipino. Ang karunungang bayan ay may iba't ibang uri, una na rito ay ang salawikain na isang patalinghagang pahayag na ginagamit ng mga matatanda noong unang panahon upang mangaral at akayin ang mga kabataan sa mabuting asal. Pangalawa ay ang mga patalinghagang pananalita na tinatawag na sawikain. Ito ay isang paraan ng pagpukaw at paghasa sa kaisipan ng tao. Nakalilibang din ito bukod sa nakadaragdag ng kaalaman. Pangatlo ay ang bugtong. Isa itong pahulaan sa pamamagitan ng paglalarawan na binubuo ng isa o dalawang taludtod na maikli at may sukat at tugma. Pang-apat naman ay ang palaisipan. Ito ay gumigising sa isipan ng tao upang bumuo ng isang kalutasan sa isang suliranin. Panlima ay ang bulong. Ito ay ang kalimitang sinasabi kapag may nadadaanang punso sa lalawigan na pinaniniwalaang tinitirhan ng mga duwende o nuno. Pang-anim ay kasabihan. Ito ay bukambibig ng mga bata at matatanda na kung tawagin sa Ingles ay Mother Goose o Nursery Rhymes. Ito ay mga tulang pambata o tugmang walang diwa o mababaw ang isinasaad na kahulugan. Pampito ay ang kawikaan na kauri ng salawikain na ang kaibahan lamang ay laging nagtataglay ng aral sa buhay (Quiambao, 2017).

May iba't ibang dahilan sa pag-aaral ng panitikan. Una na rito ay upang makilala ang kalinangang Pilipino sapagkat salamin ang panitikan sa kultura ng mamamayan. Malaman ang minanang yaman ng kaisipan at taglay na katalinuhan ng lahing pinagmulan. Pangalawa ay upang matalos na may marangal at dakilang tradisyon ang mga Pilipino na nagsisilbing patnubay sa mga impluwensiya ng ibang mga kabihasnang nanggagaling sa iba't ibang mga bansa sa mundo. Pangatlo ay upang mabatid ang mga kaisipan sa panitikan at makapagsanay upang maiwasto ang mga ito. Mahalaga talaga na malaman ang mga dahilan sa pag-aaral ng panitikan sapagkat ito ang magiging motibasyon upang mapukaw ang interes nang sa gayon ay higit itong tangkilikin at pag-aralan. 
Binanggit ni Arrogante (2007) na ang panitikan ay isang talaan ng buhay kung saan nagsisiwalat ang isang tao ng mga bagay na kaugnay ng napupuna niyang kulay ng buhay sa kanyang daigdig na kinabibilangan. Naisatitik ang mga karanasan-magaganda man o hindi. Ayon naman kay Salazar (1995) mula sa aklat ni Sauco, ang panitikan ay isang lakas na nagpapagalaw sa lipunan at isang kasangkapang makapangyarihan na maaring magpalaya sa isang ideyang nagpupumiglas upang makawala. Napakatindi talaga ng pantikan at hindi puwedeng mapigilan. May iba't ibang pananaw ang mga tao sa pagbibigay ng pakahulugan sa panitikan na mas nagpapatingkad sa kahalagahan nito sa buhay ng tao.

Ayon kay Atienza et al. (2010), na sa panitikang Filipino, inilalahad ang ideya na tunay ang isang panitikan kung nagtataglay ng walang kamatayang diwa.Ito rin ay nagpapabatid ng damdamin ng tao bilang sukli niya sa reaksiyon sa kanilang pang-araw-araw na pagsusumikap upang mabuhay at makamtan ang kaligayahan sa kanyang kapaligiran at sa kanyang pagnanais na makita ang dakilang Lumikha.

Masasalamin sa panitikan ang buhay at dahil dito mababatid ang pamumuhay at paraan ng pakikipamuhay ng mga tao sa lipunang gingalawan niya (Pangakalinawan et al., 2006). Ang isipan ay mas pinapatalas at ang puso ay pina-iibig ng mga panitikan. Ito ay bahagi ng buhay na hindi maaaring iwaglit. Ayon naman kay Tiongson (2008), ang panitikan ay kailangang maging kasangkapan upang lalong maitayo ang bandila ng pagka-Pilipino sa pamamagitan ng panulat gamit ang katutubong wika. Kung gagamitin ang katutubong wika ay mas mahimok ang mga Pilipino na tangkilikin at mahalin ang wikang sarili. Mahalaga ito upang ang isang lahi ay makilala ayon sa pag-unlad ng sariling wika sa pamamagitan ng paggamit nito. Dagdag pa ni Arrogante (2007) na makikilala ang bawat bansa sa kanilang sari-sariling kultura at sari-sariling identidad. Kaya karaniwan lamang na may sariling panitikang naglalahad ng tangi at tunay na pagkatao, pagkalahi at pagkabansa na tanda ng sariling pagkakakilanlan.

Ang mga literaturang nabanggit ay mahalaga dahil ang panitikan ay naglalahad ng katotohanan tungkol sa isang lahi at dito rin naipapahayag ang niloloob ng isang tao. Tumatalakay rin ang panitikan sa mga ideyang kinapapalooban ng impormasyon, panghihikayat, pagkakasunod-sunod ng mga pangyayari at patungkol sa pagkakakilanlan sa pamamagitan ng kultura. Ang mga nabanggit na literatura ay mahalaga pa sa kasalukuyang pag-aaral sapagkat nakatuon ang silbi at gamit ng panitikan at kultura sa ikabubuti ng sangkatauhan.

Mahalaga sa isang komunidad ang karunungang-bayan dahil hindi lang ito napagkukunan ng mga mahahalagang aral kundi ito rin ang nagiging instrumento upang hindi mawala ang mahahalagang kaugalian at kultura ng isang lugar.Ayon kay Francisco (1984) ang karunungang-bayan ay kinikilala bilang isa sa mga pinakamahalagang paraan sa pagpapamana ng mga mahalagang kaugalian o mga mabuting pag-uugali.Kinakailangan ang mahahalaga at magandang kaugalian sapagkat ayon sa pag-aaral ni Galigao (2014) isa sa mga nangungunang suliranin ng edukasyon sa kasalukuyang panahon ay ang pagkawala ng moral at espiritwal na kagandahang pag-uugali ng mga kabataan ngayon.ito ay dahil sa mga pagkalantad ng mga kabataan sa mga babasahin at panooring malalaswa,may temang puno ng violence o barumbado at materyalistiko.Kaya nga kinakailangang maipasa sa bagong henerasyon ng mga kabataan ang mga magagandang kaugalian na ipinamana $\mathrm{n}$ gating mga ninuno upang hindi ito makadagdag sa suliranin ng ating lipunan.

Sa pag-aaral ni Daniel (2013), ang bawat kultura ay koleksiyon ng mga kasabihan na kapupulutan ng talino na nagbibigay ng payo sa pamumuhay ng mga tao. Mula naman sa pag-aaral ni Timbreza (2017) ang mga katutubong wika ay kapupulutan ng mga mahahakagang kaisipan at gamit sa pakikipagtalastasan ay kapapapalooban ng artikulasyon at pormalisasyon ng mga katutubong pananaw ay nagsisilbing panghulma at modelo na pupuno sa pagkauhaw ng tao sa mga dunong at kaalaman at sa paghahanap niya ng katotohanan at kahulugan ng mga bagay-bagay o pangyayari.Ayon sa pag-aaral ni Romero (2019) ang mga salawikaing Filipino ay kinapapalooban ng mga kasabihan na nagtatalaga ng mga pamantayan,tradisyon at paniniwala sa isang komunidad.

Nang maipatupad ang CHED Memorandum Blg. 59, s.1996 na may layuning maituro ang panitikan ayon sa rehiyong kinabibilangan, mas nahimok pa na magsulat ang mga manunulat na Bikolano bilang bunga ng 
kanilang pagtanggap sa hamong ito. Ang bayan ng Irosin ay binubuo ng dawampu't walong (28) barangay na nasa gitnang bahagi ng lalawigan ng Sorsogon. Ang mga tao sa lugar na ito hanggang sa kasalukuyan ay may mga karunungang bayan pa ring pinaniniwalaan at naisasalin sa bibig ng mga susunod na henerasyon.

Ang karunungang-bayan ay nagsisislbing saligan ng pagkakakilanlan ng isang bansa na sa diwa nito ay makatutulong upang mapagyaman ang kaisipan at ugali ng isang mamamayan. Sa paraang makilala at mapayaman ng mga tao lalong-lalo na ang mga kabataan ang sariling panitikan, mas pahahalagahan pa nila ito at mamahalin bitbit ang kabatirang ito ay bahagi ng kanilang pagkatao. Kaya marapat lamang na tangkilikin at paunlarin ang mga ito.

Layunin kung gayon ng pag-aaral na ito na mailahad ang iba't ibang uri ng karunungang-bayan na bahagi na ng kultura at panitikang Irosanon na maaring gamitin na lunsaran sa pagturo upang magdulot ng kamalayan at pagpapahalaga ng mga mamamayan lalong-lalo na ang mga mag-aral ng kaniling sariling kultura at panitikan.

\subsection{Paglalahad ng suliranin}

Ang pangkalahatang layunin ng pag-aaral ay:

$>$ Malaman kung mayroon pa bang mga karunungang-bayan na nanatiling iniingatan at pinagyayaman ng mga Irosanon.

Ang mga ispisipikong layunin ay ang mga sumusunod:

$>\quad$ Matukoy ang iba’t ibang uri ng karunungang-bayan bilang bahagi ng kultura at panitikang Irosanon.

$>\quad$ Masuri ang gamit at mga bentaheng dulot ng karunungang-bayan sa buhay ng mga mamamayan.

$>\quad$ Makapagmungkahi ng gawain batay sa resulta ng pag-aaral.

\section{Metodolohiya}

Deskriptib analisis ang disenyo ng pag-aaral na ginamit ng mananaliksik sa paglikom ng mga datos na kinailangan sa pag-aaral na ito. Ang mga kalahok sa pag-aaral ay ang mga mamamayan ng bawat barangay ng bayan ng Irosin na may kaalaman sa karunungang-bayan. Binubuo ng 112 na kalahok na pinili sa pamamagitan ng alisagang halimbagay o random na mga pagsasampol (random sampling) na nagmula sa iba't ibang barangay ng bayan ng Irosin. Tseklist ang ginamit bilang instrumento sa pagkuha ng datos na kakailanganin at pakikipanayam. Ang mga nalikom na datos ay inanalisa, sinuri at binigyan ng interpretasyon gamit ang nararapat na estatistika tulad ng frequency count at pagraranggo.

\section{Mga resulta at talakayan}

Base sa nalikom na mga datos, sa Talahayanan 1 makikita ang mga iba't ibang Karunungang-bayan sa bayan ng Irosin. Ito ang mga karunungang-bayan na nananatili, iniingatan at pinagyayaman ng mga Irosanon.

\section{Talahanayan 1}

Iba't ibang karunungang-bayan sa bayan ng Irosin

\begin{tabular}{lcc}
\hline \multicolumn{1}{c}{ Karunungang-Bayan } & Kabuuan & Bahagdan \\
\hline Bugtong/Patangkudan & 49 & 1 \\
Bulong/Orasyon & 12 & 4 \\
Salawikain/Kawikaan/Sarabihon & 44 & 2 \\
Sawikain/Idyoma & 31 & 3 \\
\hline
\end{tabular}

Ipinapakita sa Talahanayan 1 ang iba't ibang karunungang-bayan na matatagpuan sa bayan ng Irosin. Base sa datos, nangunguna ang bugtong/patangkudan. May apat na pu't siyam (49) na mga bugtong/patangkudan sa 
Irosnin. Patunay lamang ito sa na mahilig ang mga taga Bikol sa mga patood o bugtong pero saying lamang at kakaunti lamang sa mga panitikang Bikolano ang nailimbag at mismo sa wikang Bikol at kalat-kalat pa. Bagamat mayaman naman talaga ang Bikol sa mga karunungang-bayan na naipasa sa mga susunod na henerasyon sa pamamagitan ng pagpasalin-salin sa bibig ng mga tao. sang pangungusap o tanong na kadalasang nilalaro ng mga batang pinoy, at ng mga nakakatanda. Ito ay may doble o nakatagong kahulugan na nilulutas bilang isang palaisipan. Ang bugtong ay gumagamit ng talinghaga, o mga metapora sa pagsasalarawan ng isang partikular na bagay o mga bagay na hulaan. Madalas itong nangangailangan ng katalinuhan o wit at maingat ng pagninilay-nilay upang mahulaan ang palaisipan o tanong. Halimbawa nito ay "Kung kailan mo pinatay, saka pa humaba ang buhay". Ano ang sagot?

Sumunod naman ang salawikain/kasabihan/sarabihon ayon sa dami na apat na pu't apat (44). Marami rami rin ang mga salawikain sa bayan ng Irosin. Ang panitikan ng Bikol ay napapalibutan ng gayuma at pagkahumaling sa napakagandang paligid nito. Ito ay nangangahulugang ang mga Bikolano ay iniuugnay sa paligid at sa kalikasan sa paglikha ng kanilang mayamang obra ng panitikan. Ang salawikain ay mga salitang maituturing na pilosopiya sapagkat ito ay may malalim na kahulugan at talaga namang matalinghaga. Hindi mga ordinaryong salita ang ginagamit sa salawikain gaya ng "Nasa Diyos ang awa, nasa tao ang gawa" at "Kung ano ang puno siya ang bunga" at marami pang iba.

Pangatlo ay ang sawikain/idyoma na may tatlong pu't isa (31). Ang kasabihang Bikolnon ay tinatawag na "Balalong" (Bicol Proverbs). Ang mga ito ay kinapapalooban ng mga mabubuting payo na hango sa tunay na buhay bilang gabay ng mga bata at matatanda sa pakikisalamuha sa matuwid na landas ng buhay. Ang sawikain o idiom sa wikang Ingles ay salita o grupo ng mga salitang patalinghaga na nagsasaad ng hindi tuwirang paglalarawan sa isang bagay, kaganapan, sitwasyon o pangyayari. Ito ay may dalang aral at kadalasan ay nagpapahiwatig ng sentimyento ng isa o grupo ng mga tao. Malalalim na salita din ang ginagamit sa sawikain gaya ng salawikain at pinapalitan ang pangkaraniwang tawag kung kaya ito ay nagiging matatalinghagang pahayag. It rin ay mga salitang lumalarawan sa isang bagay o pangyayari na kadalasan ay kumposisyunal o mahirap malaman ang tumpak na kahulugan. Mga halimbawa nito ay "Bukas ang Palad," "Abot tanaw" at marami pang iba.

Sa pangkalahatan, ang isang salawikain ay kasabihan na nagbibigay ng magandang aral sa buhay ng isang tao. Samantala, ang sawikain ay tinatawag na idyoma, isang maikling kasabihan na mayroong ibang kahulugan sa literal nitong anyo. Ang mga kasabihan naman ay mga aral na ipinasa-pasa upang magabayan ang tao buhay. Ang pagkakatulad nila ay ang pagbibigay ng aral sa mga tao. Bukod rito, nabubuo ang mga ito sa matagal na panahon. Ito ay dahil ang mga aral na dala ng mga ito ay galing pa sa mga ninuno at mga nakakatanda na may maraming karanasan sa buhay.

Pang-apat at pang huli ayon sa dami ay ang bulong/orasyon na may labing dalawa (12). Ayon sa isang kuwento, may isang misyonerong Kastila ang humanga sa pagsulat ng mga sinaunang Bikolano. Pinatutunayan lamang nito na noon pa man ay may angking talino na ang mga Bikolano na maging ang mga dayuhan ay nagbibigay-pugay. Ang bulong ay isang matandang katawagan sa orasyon ng mga sinaunang tao sa kapuluan ng Pilipinas gaya ng "Tabi tabi po, Ingkong," "Makikiraan po" at iba pa. Ito ay sinasabi kapag may nadadaanang punso sa lalawigan na pinaniniwalaang tinitirhan ng mga duwende o nuno gaya ng "Huwag magalit kaibigan, aming pinuputol lamang, ang sa ami'y napagutusan”.

Sa Talahanayan 2, ipinapakita ang gamit ng karunungang-bayan at bentahe nito sa pamumuhay ng mga mamamayan at sa kanilang pang-araw-araw na pamumuhay. Makikita rin dito ang pagkakasunod-sunod ng sagot mula sa pinakamahalagang dahilan kung paano nagagamit ang karunungang-bayan ng mga mamamayan hanggang sa pinakamaliit na dahilan ng paggamit ng karunungang-bayan. 


\section{Talahanayan 2}

Gamit ng karunungang-bayan at bentahe sa pamumuhay ng mga mamamayan

\begin{tabular}{lcc}
\hline \multicolumn{1}{c}{ Gamit ng Karunungang-Bayan } & Kabuuan & Bahagdan \\
\hline Ginagamit upang magpasintabi sa mga di-nakikitang nilalang & 19 & 7 \\
Kapupulutan ng aral sa buhay & 49 & 3.5 \\
Mas nagpapatalas ng isipan/karunungan & 49 & 3.5 \\
Nakaaalis ng stress ng isang tao & 43 & 4 \\
Nakalilibang bilang palaisipan ng mamamayan & 41 & 5 \\
Nakatutulong bilang gabay sa tamang landas ng buhay & 51 & 1 \\
Pagapapanatili ng kultura ng isang lugar & 31 & 6 \\
\hline
\end{tabular}

Ipinapakita sa talahanayan ang gamit ng karunungang-bayan at bentahe nito sa pamumuhay ng mga mamamayan. Nangunguna rito ang gamit ng karunungang-bayan na nakatutulong bilang gabay sa tamang landas ng buhay. Ayon ito sa sagot ng limam pu't isang (51) kalahok sa pag-aaral. Nakatutulong ito sa mga mga mamamayan sapagkat ito ang nagiging gabay ng mga tao sa kanilang pamumuhay. Pinatunayan ito ni Tolosa (2017) na binanggit ng mga lolo at lola na ang karunungang-bayan ay maaring maging gabay sa mga bagay na dapat maisasabuhay upang maging isang huwaran. Bilang responsableng mamamayan tungkulin ng lahat na maging modelo ng magandang gawi at isa sa maaring maging gabay ay ang pagkintal sa isipan at pagsabuhay ng mga karunungang-bayan.

Pumapangalawa sa gamit ng karunungang-bayan ay ang kapupulutan ng aral sa buhay at nagpapatalas ng karunungan at isipan na parehong nakapuwesto sa ikalawang antas. Ayon ito sa sagot ng apat na pu't siyam (49) na kalahok sa pag-aaral. Isa sa mga patunay nito ay mula kay Tolosa (2017) na ang mga karunungang-bayan ay nagpapatalas ng isipan upang mag-isip at mabigyang-kahulugan ang mahahalagang kaisipang nakapaloob dito o ang mga salitang inilalarawan. Ang karunungang-bayan ay isa sa puwedeng mag-ehersisyo ng isipan sapagkat kapag nakabasa ang tao nito ay magdudulot ito ng pag-andar ng isipan upang ukilkilin ang natatagong dunong o ideya mula dito. Isa itong paraan ng kritisismo o pagsusuri.

Pang-apat sa mga gamit ng karunungang-bayan ay nakakaalis ng stress o pagod ng isang tao. Ayon naman ito sa sagot ng apat na pu't tatlong (43) kalahok sa pag-aaral. Nakakatanggal ng stress ang mga karunungang-bayan lalo na kung ito ay ginagamit bilang pang-alis ng pagkabagot at pang-alis ng problema sa buhay ng mga tao. Ang pagtawa nang madalas at paglibang sa sarili ay nakatutulong upang palakasin ang immune system ng isang tao. Kailangan ring mag-isip ng mga positibong bagay ukol sa sarili at sa kapaligiran at turuan ang sarili na may mga bagay na sadyang di-kayang baguhin.

Panlima ang nakalilibang bilang palaisipan ng mga mamamayan. Ayon naman ito sa sagot ng tatlumpung isang (41) mga kalahok sa pag-aaral. Sa pagsagot sa mga palaisipan tulad ng bugtong ay maaaring malibang ang isang tao at panandaliang malimutan ang mga suliranin sa buhay na nararanasan. Bago pa dumating ang mga dayuhan ay may iba't ibang karunungang-bayan na ang mga ninuno ng mga Pilipino na ginawang libangan sa mga tahanan tulad ng pagkukuwento ng mga alamat, kuwentong-bayan, epiko, awiting-bayan at marami pang iba pa.

Pang-anim sa mga gamit ng karunungang-bayan ay ang pagpapanatili ng kultura ng isang lugar. Ayon ito sa sagot ng tatlong pu't isang (31) kalahok sa pag-aaral. Isa sa mga kahalagahan ng panitikan ay upang mabatid ang mga sinaunang kaugalian, tradisyon at kultura. Kaakibat ng paglago ng kultura ng isang lugar ay ang paglago rin ng panitikan na maaring malaman kung parehong bibigyan ng pansin.

Pampito sa mga gamit ng karunungang-bayan ay upang magpasintabi sa mga di-nakikitang nilalang. Ayon ito sa sagot ng labing siyam (19) na kalahok sa pag-aaral. Ang bulong ay ginagamit bilang pagbibigay-galang o pasintabi. Dahil sa paniniwala ng mga ninunong Pilipino sa mga nilalang na may kababalaghan tulad ng mga engkanto, nuno sa punso at iba pa. Nabuo ang bulong bilang pagpapasintabi o pagbibigay-galang sa mga hindi nakikitang nilalang. 


\section{Talahanayan 3}

Mga halimbawa ng karunungang-bayan na matatagpuan sa bayan ng Irosin

\section{Halimbawa ng Karunungang-Bayan}

\section{SALAWIKAIN/KASABIHAN/KAWI-KAAN (SARABIHON)}

Barangay San Julian, Gabao, Macawayan, Patag, Gulang-Gulang, Tinampo, Batang, Salvacion, Casini, Liang, Tabon-Tabon, Bacolod

A

"Aanhon pa an sakate kun gadan na ining kabayo ni Jose"

-Ano pa raw ang gagawin sa bagay na kinakailangan kung wala na ang makikinabang nito.

"An dilang matarom,kun nakalugad hararom"

-Ang dila raw ng isang taong matabil at walang preno magsalita ng masasakit na salita ay puwede raw makasakit ng loob na maaaring matagal maghilom.

1

"An may isinang-at sa paga,may gagawadon pagkaaga"

-Ang tao raw na may itinabing bagay o inimpok pag dating ng umaga o araw ay may makukuha kung kinakailangan na.

"An tawo na duduso,bisyoso"

-Ang isang tao daw na iyon palagi ang ginagawa ay palaging nagdududa sa gawain din ng ibang tao.

"An tawo na matuturugon,mamamatayun"

-Ang isang tao raw na tamad at palaging natutulog sa pansitan ay malaki ang posibilidad na mamatay sa kahirapan o maghirap sa hinaharap.

"An tawong hugakon warang aabuton"

-Ang tao raw na tamad ay malaki ang posibilidad na walang maabot na pangarap sa buhay sa hinaharap.

$2 \boldsymbol{B}$

"Basta kuwarta,burarat an mata"

-Pag dating sa pera o usapang pera karamihan sa mga tao ay interesado o makikinig.

$D$

"Dai nin langit kun dai nin sakit"

-Ang buhay daw ng tao ay walang ginhawa kung hindi ka muna dumaan sa mga pagsubok o paghihirap.

$3 \mathrm{H}$

"Higos tios, hugak tigbak"

-Ang tao raw na masipag ay may paghihirap ngunit kapalit nito ay ginhawa samantalang ang taong tamad daw ay malaki ang posibilidad na mamatay sa hinaharap na dahil sa katamaran ay naghirap.

$\boldsymbol{K}$

"Kun an bituun nan bulan nagkataningan,igwang mababadusan"

-Matandang kasabihan na pinaniniwalaang kapag nagkataong magkatabi ang buwan at bituin ay merong magtatanan at mabubuntis.

4

"Kun nano an habo mo himoon sa sadiri mo,deri mo pag-himoon sa iba na tawo"

-Kung ano daw ang ayaw mong gawin sa sarili mo ay huwag mo raw gagawin sa ibang tao.

"Kun sin-o an an nagbunay siya an nagkutak"

-Kung sino daw ang unang taong nagsalita ay siya raw ang may gawa ng isang bagay.

$N$

"Natatago an kayamanan, dai an kapobrehan"

-Ang kayamanan daw ng isang tao ay pwede mong itago pero ang kahirapan ay hindi maikukubli.

\section{$5 \quad P$}

"Pag an tawo maparanga,daghan an minahanga"

-Kapag ang isang tao raw ay masipag malaki raw ang posibilidad na marami ang humanga sa kanyang kasipagan.

"Pag binato ka sin bato,batuhun mo sin tinapay"

"Pag sinapuyong ka sa too mong pandok,ihatag mo pa an wala"

-Kapag ginawan ka raw ng masama ng iyong kapawa huwag kang gaganti bagkus gawan mo ang taong ito ng mabuti.

"Pag may intago may kukuhaon"

-Ang taong may gawi daw ng pag-iimpok sa oras ng kanyang pangangailangan ay may makukuha para sa anumang paggagastusan.

6

"Pakadto ka pa lang,pabalik na ako"

-Ang paksa ay tungkol sa pagpapaalala ng isang mas may karanasan o may kaalaman na matanda sa isang walang karanasan at walang kaalaman na mas bata.

"Para sa imo siya bakting pero para sa ako siya birhen"

-Ang kagandahan ng isang tao ay nakadepende sa tumitingin.

"Piso gana barko"

-Ang kaliitan ng halaga ng isang bagay ay may kapalit na malaking halaga. S

"Sa alpog ka hali,sa alpog ka man gihapon mauli"

-Ang tao raw dahil sa alikabok nanggaling sa alikabok din siya babalik.

$7 \quad U$

"Ubos-ubos biyaya,pagkaaga nakatunganga"

-Ang tao raw na gastador pagdating ng bukas ay wala ng pera o pag-aari. 
Llaneta, R. N.

Talahanayan 3 ...pagpapatuloy

Halimbawa ng Karunungang-Bayan

BUGTONG/PALAISIPAN (PATANGKUDAN)

Barangay San Juan, San Pedro, San Agustin, Tongdol, Mapaso, Bolos, Liang, Bagsangan, Monbon, Sto.Domingo, Cawayan, Macawa-yan,

"An bata nakaingkod na, an ina naggagapang pa" -Kalabasa

"An bobong kawali,ang tuhod barari" -Alimasag

"An payong san agta,lain nababasa" -Dahon ng gabi

"Arado an inutan, witwit an likudan" -Baboy

9 B

"Balay ko sa Masbate,saro-saro an harigi" -Payong/Kabute

"Balay ni San Gabriel,palibot sin badil" -Papaya

"Balay ni Sta.Ana,palibot sin espada" -Pinya

"Binotong ko si balagon,naribok si maghapon" -Kampana

"Burak mun-a an dapat himuon,bago kaunon" -Saging

10 D

"Didi ko tinanom,didto tuminubo" -Flashlight

$\mathbf{H}$

"Hilaw,tubod naglalakaw" -Kalabaw

"Kalibkib san lobi,nag-iidos kun gab-i" -Buwan

11

"Kapoti an ikog ko,kay mabuso ako" -Tabo

"Kun basog tindog,kun gutom lup-og” -Sako

M

"Magakamanghod na prinsesa,bagan an mga negra" -Duhat

"Manay mo,manay ko,manay nato intiro" -Atis

“Mapa-sa-tubig,mapa-sa-kalayo,dai naano" -Anino

"May ngipon wara hiwa,may siki wara kamot" -Kudkuran

12

"May alaga ako na manok,abot didi an tiraok" -Kulog

"Nagsiyak si San Pedro,hilinga ta naglalaad an tingo" -Kulog at kidlat

"Nagtago si Pedro,nakaluwas an ulo" -Pako

"Pag-agi ni Kuwaw, hinigop an mga sabaw" -Bahaghari

13

"Pag-agi san Amerikano,nagkaburuhay an mga tawo" -Umaga

"Pag-agi san Negro, nagkamaratay an mga tawo" -Gabi

“Saday pa si Biyay,maaram na magpilay-pilay" - -Alimasag

"Saday pa si Nene,maaram na magtahi" -Gagamba

"Sa ibabaw kapatagan,sa irarom kakahuyan" -Posporo

14

"Sa ibabaw tul-ang,biribid an laman" - -Alimasag

"Saro an nilaugan,tolo an linuwasan" -Kamiseta

"Sira ko sa Mariveles,sa irarom an kariskis" -Sili

T

"Tubig sa Rikandikan,dai nauuranan" -Sabaw ng Niyog/Buko

$15 \mathbf{U}$

"Upat katawo,saro an kalo" -Bahay 
Talahanayan 3 ...pagpapatuloy

Halimbawa ng Karunungang-Bayan BULONG (ORASYON)

Barangay San Julian, Tabon-Tabon, Patag, Carriedo, Batang, Casini, Gabao, Bulawan, Gulang-Gulang, Sto.Domingo, Cawayan, Macawa-yan, Buenavista, Bacolod

A

Arot pagutpot,nakasamo sa ipot!

(Ang kalbo ay nakahalo sa ipot)

B

Bagyo,bagyo pantay kabayo!

H

Huyop-huyop sagalpot salbahe an naghapot!

(Pag-ihip ng hangin ang nagtatanong ay salbahe raw)

$17 \mathbf{P}$

Pira nunot,pira sibang,pira gabos!

(Puwera usog,puwera lahat)

$\mathbf{T}$

Taba,tubol nakasakay sa bagol!

(Ang mataba ay nakasakay sa bao ng niyog)

Tabi,tabi makiagi!

(Tabi tabi po makikiraan po)

SAWIKAIN/IDYOMA (TALINGHAGA)

Barangay San Julian, Tabon-Tabon, Patag, Carriedo, Batang, Casini, Gabao, Bulawan, Gulang-Gulang, Sto.Domingo, Cawayan, Macawa-yan, Buenavista, Bacolod

$$
\text { A }
$$

Amoy Pinipig

-mabango

Amoy Tsiko

-lasing

$\mathbf{L}$

Luho an bulsa

-walang pera

$19 \mathrm{M}$

Magub-at an lawas

-tamad

Makatol an kamot

-magnanakaw

Makatol na babaye

-malandi

Makudat an ulo/Matugas an ulo

-suwail

Makulog an ulo

-suwail/pasaway

Marugi an lawas

-marunong sumayaw

Matampol an utak/Wara sulod an ulo

-bobo,mahina ang ulo

$\mathbf{T}$

Tawo sa likod

-mga engkanto o elemento

Masasalamin sa Talahanayan 3 ang iba't ibang halimbawa ng karunungang-bayan na matatagpuan sa bayan ng Irosin. Una na rito ay ang salawikain, kasabihan, kawikaaan at tinatawag ng mga Irosanon na "sarabihon" na may 21 na halimbawa. Ayon sa www.notes-tips.blogspot.com, ang mga salawikain ay mga pangungusap na maiiksi lamang ngunit punong-puno ng kahulugan.may layunin din itong magbigay ng patnubay sa ating pang-araw-araw na pamumuhay. Sumasalamin din ito sa kultura at tardisyon nating mga Pilipino. Ginagamit ito $\mathrm{n}$ gating mga ninuno noong unang panahon bilang gabay upang maging mabuting mamamayan ng isang lugar at bilang paggalang sa pamana ng lahi na mga tradisyon at kultura. Ikalawa sa mga halimbawa ng karunungang-bayan ay ang mga bugtong at palaisipan na tinatawag ng mga Irosanon na "patangkudan" na may 32 na halimbawa. Mula naman sa www.tl.m.wikipedia.org ang bugtong, pahulaan, o patuturan ay isang pangungusap o tanong na may doble o nakatagong kahulugan na nilulutas bilang isang palaisipan. May dalawang uri ang bugtong:mga talingghaga o enigma, bagaman tinatawag ding enigma ang bugtong, mga suliraning ipinapahayag sa isang metapora o ma-alegoryang wika na nangangailangan ng katalinuhan at maingat 
na pagninilay-nilay para sa kalutasan, at mga palaisipan,mga tanong na umaasa sa dulot na patudyong gamit sa tanong o sagot.

Ang mga bugtong ay libanagan na ating mga ninuno na nangangailanagn ng higit na pag-iisip at pag-aanalisa upang mahulaan ang tinutukoy ng pangungusap o tanong.Ikatlo sa mga halimbawa ng karunungang-bayan ay ang bulong na tinatawag ng mga Irosanon na "orasyon" na may 6 na halimbawa. Nabanggit naman mula sa www.tl.m.wikipedia.org ang bulong ay isang panalangin na binuhay sa pagnanais na makamtan ang isang pangyayari o pagbabago sa hinaharap na mga pangyayari sa kapalaran.karaniwan ang bulong ay ginagamit sa panggagamot ngunit pwede rin itong gamiting pang-akit ng mga bagay na nais mangyari at pantaboy sa mga di nais na mga bagay o pangyayari. Ikaapat sa halimbawa ng karunungang-bayan ay ang sawikain o idyoma o tintawag ng mga Irosanon na "talingghaga" na may 12 na halimbawa. Ayon ulit sa www.tl.m.wikipedia.org ang isang sawikain o idyoma ay isang pagpapahayag na ang kahulugan ay hindi komposisyunal sa ibang salita, hindi binubuo ng tumpak na kahulugan ang mga kanya-kanyang salita na nabuo.Ito ay di-tuwirang pagbibigay kahulugan at pagpapakita ng kaisipan at kaugalian ng isang lugar. Ginagamit ang mga idyoma bilang mga pangakaisipang salita na hindi direktang tinutukoy ang ibig ipakahulugan sa literal na kahulugan.

\subsection{Nabuo batay sa resulta ng pag-aaral}

Ang nabuo dahil sa pag-aaral na ito ay isang aklat na pinamagatang Karunungang-bayang Irosanon. Ito ay naglalaman ng mga karunungang-bayan mula sa mga taga-Irosin bilang ambag sa Irosanong kultura at panitikan.

\section{Kongklusyon}

Batay sa natuklasan, nabuo ang sumusunod na kongklusyon:

$>\quad$ Ang iba't ibang karunungang-bayan sa bayan ng Irosin na nananatili pa at iniiingatan ay ang mga sumusunod: (1) Bugtong/palaisipan o patangkudan ang unang uri ng karunungang-bayan na may mga paglalarawan o matalinghagang salitang ginagamit; (2) Salawikain/kawikaan o sarabihon na nagpapahayag ng mahalagang kaisipan upang maging gabay ng mga mamamayan sa kanilang pang-araw-araw na buhay tungo sa matuwid na landas; (3) Sawikain/idyoma na ginagamit pa rin ng mga matatanda sa pangangaral sa mga tao upang tumungo rin sa matuwid na landas; (4) Bulong o orasyon na isang uri rin ng karunungang-bayan na ginagamit upang manudyo o manukso sa mga batang kalaro o magpasintabi sa mga nilalang sa paligid na pinaniniwalaang mga elemento o engkanto.

$>\quad$ Ang iba't ibang gamit at bentahe ng karunungang-bayan mula sa iba't ibang barangay ng bayan ng Irosin ay ang mga sumusunod: (1) Nakatutulong ang mga karunungang-bayan bilang gabay sa tamang landas ng buhay; (2) Kapupulutan ang mga ito ng aral sa buhay at mas nagpapatalas ng isipan/karunungan; (3) Nakaalis ng stress ng isang tao; (4) Nakalilibang bilang palaisipan ng mga mamamayan; (5) Nakatutulong upang mapanatili ang kultura ng isang lugar; at (6) Ginagamit upang magpasintabi sa mga di-nakikitang nilalang.

\section{Rekomendasyon}

Batay sa resulta ng pag-aaral na ito nabuo ang mga sumusunod na rekomendasyon:

$>\quad$ Hikayatin ang mga mag-aaral at mga mamamayan sa komunidad na magbasa tungkol sa sariling kultura sa pamamagitan ng mga panitikang naisulat upang maikintil ito sa kanilang kamalayan nang hindi ito mamatay bagkus ay patuloy na payabungin at linangin pa.

$>\quad$ Gamitin ang mga panitikang may temang kultural bilang lunsaran sa pagtuturo upang matapos mag-iwan ng kabatiran sa isipan ng mga kabataan ay mapahalagahan nila ito. 
$>\quad$ Ang lokal na pamahalaan ay mahikayat na maglaan ng programa at mga patimpalak na pupukaw sa interes ng mga mamayan na pahalagahan ang sariling kultura.

$>\quad$ Pukawin ang damdaming kultural sa pamamagitan ng pagbabasa sa akda na naisulat mula sa pag-aaral na ito.

> Magsagawa ng pag-aaral na may kaugnayan sa kasalukuyang pananaliksik nang sa gayon ay mapalalim pa ang pagtuklas sa mga kaalamang makatutulong upang mas mapahalagahan ang sariling kultura.

Pagkilala - Ang pag-aaral na ito ay hindi magkakaroon ng katuparan kung hindi dahil sa tulong at suporta ng mga taong naniniwala sa layunin ng mananaliksik, kung kaya't taus pusong nagpapasalamat ang mananaliksik sa mga sumusunod: Sa paaralang Gradwado, Sorsogon State College sa pagbibigay sa mananaliksik na makapag-aral sa institusyong ito. Dr. Helen R. Lara, Pangulo, Sorsogon State College sa patuloy na pagbibigay ng suporta sa paaralang Gradwado. Dr. Gerry A. Carretero, Dekano, Paaralang Gradwado, Sorosgon State College, sa pagsuporta sa paghikayat sa mga mag-aaral na magpatuloy sa pagsasagawa ng pananaliksik. Dr. Felisa D. Marbella, Tagapayo, sa kanyang pagiging matiyaga, maalalahanin, at walang sawang pagpapaalala, gabay at tulong sa mananaliksik at ng pagbigay ng panahon at mungkahi para sa ikauunlad ng pag-aaral na ito. Sa lahat ng mga tao sa bayan ng Irosin na tumulong upang maisakatuparan ang pag-aaral na ito. Sa aking mga minamahal na magulang Raul F. Llaneta at Lilia N. Llaneta, maybahay na si Maricel at anak na si Railey Axel. Higit sa lahat sa Ina ng Walang Hanggang Saklolo at sa Poong Maykapal na walang sawang gumabay at tumulong anuman ang mga balakid at pagsubok sa pag-aaral na ito. Maraming salamat po sa inyong lahat! R.N.L.

\section{Mga Sanggunian}

Arrogante, J. A. (2007). Filipino academic commission [Komisyon sa akademikong Filipino]. .Unlad Publishing House.

Atienza, O. L., et al. (1984). Philippine literature [Panitikang Pilipino]. Katha Publishing.

Daniel, C. A. (2013). Proverbs. Hekima Review, 49.

Francisco, J. R. (1984). Fifth Philippine folklore. University of the Philippine Press.

Galigao, R. P. (2014). Cebuano folklore and the embedded Filipino values. UV Journal of Research, 8(1), 257-267.

Pangakalinawan, L. G., et al. (2006). Literature of different regions [Panitikan sa iba't ibang rehiyon]. Mutya Publishing House.

Quiambao (2017). Folklore [Karunungang-bayan].Retrieved from http://www.slideshare.net.html

Romero, R. B. (2019). Unoni: A textual analysis of Ybanag proverbs. The Asian ESP, 15, 344-362.

Rubin, L. T., et al. (2006). Literature in the Philippines [Panitikan sa Pilipinas]. Rex Bookstore.

Sauco, C. P. (n.d.). Philippine literature, regional [Panitikan ng Pilipinas, panrehiyon]. Goodwill Trading Co.

Timbreza, F. T. (2017). Articulation of indigenous philosophy [Artikulasyon ng katutubong pilosopiya]. Malay, 30(1), 86-98.

Tiongson, W. R. (2007). Tourism management. Office of Cultural Research.

Tolosa (2017). Folklore [Karunungang-bayan]. Retrieved from http://www.prezi.com.html

Uriarte. (2019). Reason for studying literature [Dahilan sa pag-aaral ng panitikan]. http://www.panitikankarunungangbayanblogspot.com.html 
Llaneta, R. N. 